\title{
Reducing potentially inappropriate medications in palliative cancer patients: evidence to support deprescribing approaches
}

\author{
Julian Lindsay • Michael Dooley • Jennifer Martin • \\ Michael Fay • Alison Kearney • Michael Barras
}

Received: 23 July 2013 / Accepted: 5 December 2013 / Published online: 21 December 2013

(C) Springer-Verlag Berlin Heidelberg 2013

\begin{abstract}
Objectives Cancer patients who have transitioned from curative intent chemotherapy or radiotherapy to palliative therapy have limited life expectancies. Due to this, medications for primary and secondary prevention or those with no short-term benefit are potentially inappropriate medicines in this patient group. These medications often have potentially harmful profiles, increasing the patient's adverse drug events, pill burden, and medication costs. This review evaluates the most current evidence to assess the outcomes and potential methods used for identifying and ceasing potentially inappropriate medications (PIMs) in palliative cancer patients.

Methods A systematic review of the literature was conducted using the databases Ovid MEDLINE, PubMed, EMBASE, IPA, and CINAHL.

Results Of the 51 articles examined in detail, three studies relating to cancer have been evaluated. In these retrospective and cross-sectional studies, the incidence of PIMs was shown in approximately $20 \%$ of patients, although the studies were inconsistent. In addition, six studies were identified that demonstrated the evidence in other population groups; these studies have been selected to establish the evidence in large-scale retrospective studies, prospective cross-sectional studies, both
\end{abstract}

J. Lindsay $(\bowtie) \cdot$ M. Fay $\cdot$ A. Kearney $\cdot$ M. Barras

Royal Brisbane and Women's Hospital, Brisbane, Queensland,

Australia

e-mail: julianlindsay1@gmail.com

J. Lindsay $\cdot$ M. Dooley $\cdot$ M. Barras

Monash University, Melbourne, Victoria, Australia

M. Dooley

Pharmacy Department, Alfred Health, Melbourne, Victoria, Australia

J. Martin · M. Fay $\cdot$ M. Barras

Princess Alexandra Hospital, The University of Queensland Medical

School, Brisbane, Queensland, Australia demonstrating the prevalence of PIMs, as well as the outcomes of ceasing PIMs.

Conclusion There is evidence that PIMs are commonly prescribed in palliative care patients. There are no studies that have identified the impact of ceasing PIMS in this setting. Published tools and implemented strategies have focused on the elderly populations. Further research is warranted in establishing clear guidelines for the identification of PIMs in palliative cancer patients as well as interventional studies assessing the outcomes of ceasing PIMs in these patients.

Keywords Deprescribing · Drug therapy $\cdot$ Potentially inappropriate medications $\cdot$ Medical futility $\cdot$ Neoplasms . Cancer

\section{Background}

Cancer patients who have transitioned from curative intent chemotherapy or radiotherapy to palliative therapy have a poor prognosis, with estimated life expectancies commonly less than 6 months [1]. It is therefore possible that medications for primary and secondary prevention of other illnesses (based on a non palliative life expectancy) [2] or those with no shortterm benefit are potentially inappropriate for these patients. These medications often have potentially harmful profiles, increasing the patient's adverse drug events, pill burden, and medication costs [3]. Despite this, the incidence of polypharmacy in palliative cancer patients is common. It has been reported that metastatic cancer patients take an average of seven medications, of which preventive medications account for $81 \%$ [4].

In recent years there has been a growing level of evidence [5] for "deprescribing," a term used to describe the rationalization of medicines that provide a limited benefit in patients, due to changing medical and patient factors over time [6]. 
Often, this requires an extensive review of a patient's individual medications in which clinicians consider the goals of care, treatment targets, and the potential risks and benefits of medications, as well as the patient's remaining life expectancy [7]. An effective approach in deprescribing for a particular patient group is to consider the cessation of "potentially inappropriate medications" (PIMs). In geriatrics, PIMs are classified as medications or medication classes that should generally be avoided in persons 65 years or older because they are either ineffective or they pose unnecessarily high risks for older persons and a safer alternative is available [8]. Guidelines such as Beers Criteria [9] and Screening Tool of Older Person's Prescriptions (STOPP)/Screening Tool to Alert doctors to Right Treatment (START) Criteria [10] have been developed to assist in the identification of PIMs in the geriatric population and have been used extensively since their development [11]. Unlike that population, however, palliative cancer patients often experience distressing symptoms due to their malignancy and receive numerous appropriate medications to treat complications from treatment, cancer-related symptoms, and comorbid illnesses [12].

This review aims to evaluate both the incidence, as well as any methods used to identify PIMs in palliative cancer patients. In addition, this review aims for evidence of patient outcomes in ceasing PIMs in this group.

\section{Methods}

A literature search was conducted in April 2013 using the databases Ovid MEDLINE, PubMed, EMBASE, IPA, and CINAHL. Expanded MeSH terms were used for Medical Futility/, Drug Therapy/and Neoplasm/as well as terms: potentially inappropriate medication, potentially inappropriate prescribing, deprescribing, futility, rationalization, cancer, oncology, and palliative. Boolean operators "or" and "and" were used to focus the search. Inclusion criteria consisted of all human trials irrespective of age, sex, or ethnicity.

\section{Results}

An electronic search identified 315 potentially relevant citations using the search terms described. After limiting the search to Meta analyses, randomized controlled trials (RCTs) or clinical trials, 51 citations were identified. Of these, 1 metaanalysis, 13 randomized controlled trials, and 37 retrospective or prospective trials were assessed. There were only three trials relating to cancer, which have all been evaluated. In addition, six studies that described the incidence and outcomes of PIMs in other population groups were selected by the primary author with consensus from all other co-authors. Five studies evaluated the evidence using large-scale retrospective or prospective cross-sectional designs and quantified the prevalence of PIMs. One randomized controlled trial demonstrated the outcomes of ceasing PIMs. The rationale for assessing other patient groups, such as the elderly, was to determine the level of evidence and outcomes associated with the introduction of criteria and guidelines. This was prompted by the obvious gap in knowledge for palliative oncology patients, as demonstrated by the lack of publications in this group. The included articles were selected based on the date of publication, the number of participants, and lack of confounding factors. Table 1 summarizes the nine trials and their key factors.

Potentially inappropriate medications in advanced cancer

Riechelmann et al. [13] retrospectively reviewed the charts of 372 consecutive ambulatory patients with advanced cancer who were receiving supportive care exclusively at a single center in Canada. The study found that 82 patients $(22 \%)$ were using at least one "futile medication" or PIM, defined as "unnecessary" (when no short-term benefit to patients with respect to survival, quality of life, or symptom control was anticipated) or "duplicate" (two or more drugs from the same pharmacological class). The median survival of patients was approximately 2 months; however, patients still receiving cancer-directed therapy were excluded. As no medications were ceased, the outcomes of ceasing medications could not be measured; however, the study was useful in providing a snapshot of the proportion of cancer patients taking at least one PIM as well as the types of medications prescribed. The most common PIMs were statins (56\%) and multivitamins (30\%). Although the most common comorbidities were cardiovascular disease (36\%) and diabetes (14\%), antihypertensive and hypoglycemic agents could not be considered to be PIM as the investigators were not able to reliably determine retrospectively blood pressure and serum glucose levels. The authors concluded that prospective studies in the community setting and population-based analyses are warranted to evaluate the clinical and economic impact of futile medication use in oncology practice.

Fede et al. [1] conducted a cross-sectional survey of unnecessary medication use among 87 ambulatory patients with advanced solid tumors and terminal disease who were being treated in Brazil. The group found that $24 \%$ of patients were taking at least one PIM, the most common being gastric protectors. Terminal disease was defined as having a life expectancy of less than 6 months as predicted by the patient's oncologist. The investigators also considered any patients receiving second-line chemotherapy for metastatic solid tumors, with the exception of breast cancer. The study group used a self-designed criteria to assess patients' medications and identify PIMs. The criteria consisted of ceasing; statins, if there was no cardiovascular event in the prior 12 months; 
Table 1 Key factors of the identified trials

\begin{tabular}{|c|c|c|c|c|c|c|}
\hline & Number & Population type & Study type & $\begin{array}{l}\% n>1 \\
\text { PIM }\end{array}$ & $\begin{array}{l}\text { Criteria for } \\
\text { discontinuation }\end{array}$ & $\begin{array}{l}\text { Was an outcome } \\
\text { measured? }\end{array}$ \\
\hline Riechelmann et al. 2009 [13] & 372 & Cancer patients & Retrospective audit & $22 \%$ & Medical officer assessment & No \\
\hline Fede et al. $2011[1]$ & 87 & Cancer patients & Cross-sectional survey & $24 \%$ & 5-point table & No \\
\hline Todd et al. 2013 [14] & 20 & Lung cancer patients & Retrospective audit & $95 \%$ & Medical team assessment & No \\
\hline Nyborg et al. 2012 [19] & 445,900 & $>70$ years & Retrospective audit & $34.8 \%$ & NORGEP criteria & No \\
\hline Buck et al. 2009 [15] & 61,251 & $>65$ years & Cross-sectional audit & $23 \%$ & Beers Criteria/Zhan Criteria & No \\
\hline Bongue et al. 2011 [17] & 35,259 & $>75$ years & Cross-sectional audit & $35.6 \%$ & French PIM list & No \\
\hline Gallagher et al. 2011 (May) [20] & 900 & $>65$ years & Prospective audit & $51.3 \%$ & STOPP/START algorithm & No \\
\hline Garfinkel et al. 2010 [21] & 70 & $\begin{array}{l}\text { "Elderly" referred } \\
\text { patients }\end{array}$ & Cohort study & $58 \%$ & $\begin{array}{l}\text { The good palliative-geriatric } \\
\text { practice algorithm }\end{array}$ & Yes \\
\hline Gallagher et al. 2011 (June) [10] & 382 & $>65$ years & RCT & $58.4 \%$ & STOPP/START algorithm & Yes \\
\hline
\end{tabular}

gastric protectors, without a medical history of gastrointestinal bleeding, peptic ulcer, gastritis, or known chronic use (more than 30 days) of anti-inflammatory agents (steroids and nonsteroid); antihypertensives, if the patient was taking an antihypertensive agent and presented with arterial blood pressure $<90 / 60 \mathrm{mmHg}$ at the time of last consultation and symptoms of hypotension; anti-diabetic medications in patients who were taking an anti-diabetic agent and presented with either a single measure of fasting glucose $<50 \mathrm{mg} / \mathrm{dl}$ within 4 weeks of consultation or reported symptoms of hypoglycemia and had a fast glucose test below lower normal limit; or any other medication with no clear medical indication identified [1]. As the study was a survey, no medications were ceased and therefore, like Reichmann et al., the patient outcomes and benefits cannot be measured. At least one medication was perceived as an economic burden to $44 \%$ of patients, and $26 \%$ of patients reported a drug-related adverse event, with diarrhea and dry mouth being the most common. The study also identified that a lack of medication reconciliation and a Charlson Comorbidity Index $\leq 1$ [1] were significant predictors of unnecessary medication use. The study made recommendations that health care professionals providing care for cancer patients make medication reconciliation routine, and that further research is warranted to further understand reasons for unnecessary medication use in oncology and to elucidate the economic impact of such use [1].

Recently, Todd et al. [14] retrospectively assessed the prevalence of inappropriate medication in patients taking erlotinib for the treatment of advanced non-small cell lung cancer in a multicenter study across three sites in the North of England. Of the 20 patients assessed, 19 (95\%) were taking medications that were classified as inappropriate by the clinical team. Medications were considered inappropriate after a peer assessment by a clinical team consisting of a pharmacist, nurse, and oncologist, who considered factors such as remaining life expectancy of the patient, time until benefit of the treatment, as well as goals of care and treatment targets. The study found that the most commonly prescribed PIMs were proton pump inhibitors (55 \% of patients), as well as antiplatelet $(30 \%)$ and lipid-regulating drugs (15\%) used for secondary prevention of cardiovascular events. The authors recommended that clear guidelines are needed on discontinuing inappropriate medication in patients with advanced lung cancer.

Potentially inappropriate medications in geriatrics

In 2009, Buck et al. [15] conducted the first published US multicenter study that measured the prevalence of PIMs in outpatient practices using data from electronic health records. The study was able to screen data from 61,251 current patients over the age of 65 years for PIMs using both the Beers [9] and Zhan [16] criteria, independently of diagnoses or conditions. The study showed an average of $23 \%$ of patients had $>1$ PIM using the Beers Criteria and $17 \%$ of patients had $>1$ PIM using the Zhan Criteria. The study also found that gender, polypharmacy, and the number of primary care visits were significantly associated with PIM prescribing.

Similarly, Bongue et al. [17] conducted a cross-sectional study from a sample of patient data extracted from electronic health records of the French National Insurance Healthcare System database, which covers $80 \%$ of the general population. Drugs reimbursed to all patients aged 75 years and over for a 12-month period between 2007 and 2008 were analyzed. The study identified 35,259 patients who received at least one reimbursed medicine of which 18,864 (53.5\%) received at least one PIM. PIMs were identified based on the French PIM list developed by a consensus panel [18]. This list includes medications in 34 categories, which are classified as drugs with an unfavorable benefit/risk ratio; drugs with questionable efficacy; or drugs with both an unfavorable benefit/risk ratio and a questionable efficacy. Three medication classes of PIMs were most prevalent: cerebral vasodilators, drugs with antimuscarinic properties, and long half-life benzodiazepines. The study also found correlations between PIMs and the patient demographic, as well as patient gender and age. 
More recently, Nyborg et al. [19] conducted a pharmacoepidemiological retrospective cross-sectional survey, which used data from the Norwegian Prescription Database that includes all prescriptions written by doctors in Norway that were dispensed by pharmacies to home-dwelling elderly of $\geq 70$ years in 2008 . A total of $11,491,065$ prescriptions for 445,900 individuals were assessed, which accounted for $88.3 \%$ of the Norwegian population $\geq 70$ years. The study identified that $155,341(34.8 \%)$ individuals received one or more PIM; 64,331 (14\%) received two or more; and 3,360 $(0.8 \%)$ received five or more PIMs. To assess PIMs, the study used a Norwegian list of criteria that was recently developed for general practice called the Norwegian General Practice (NORGEP) Criteria. The NORGEP uses a list of explicit criteria for pharmacological inappropriateness, consisting of 36 statements, 21 regarding single drugs and 15 regarding drug-drug combinations. The list was based on the Beers Criteria and was derived through a three-round Delphi consensus process involving 48 specialists in geriatrics, clinical pharmacology, and family medicine. The study also found significant correlations between the likelihood of receiving a PIM and age, sex, and the number of prescribers involved with a patient. A limitation of the study was that information on medications dispensed to individuals residing in institutions (e.g., hospitals and nursing homes) or medication sold over the counter was not captured by the database and therefore not evaluated.

In 2011, Gallagher et al. [20] conducted a prospective study, of 900 patients aged $\geq 65$ years who were admitted with acute illness to the acute geriatric medicine units of six teaching hospitals throughout Europe. Using both the STOPP/ START and Beers Criteria to assess PIMs, the study identified that $51.3 \%$ of patients received at least one PIM according to STOPP Criteria and $30.4 \%$ received at least one PIM according to Beers criteria. The most frequently encountered STOPP Criteria included benzodiazepines and neuroleptics in patients at risk of recurrent falls, duplicate drug class prescriptions, prolonged use of high-dose proton pump inhibitors, and prolonged use of long-acting benzodiazepines. The study showed a significant association between the prescription of a STOPP-listed PIM and female gender, cognitive impairment, and increasing numbers of medications, while Beerslisted PIMs were significantly associated with recent hospitalization and increasing numbers of prescribed medications. An advantage of the prospective study design is that Beers' "considering diagnosis" criteria could be used, which was shown to account for $59 \%$ of the overall Beers Criteria PIM prevalence rate in the study. The authors demonstrated that retrospective studies that limit their assessment of PIM use to Beers' "independent of diagnosis" criteria alone may substantially under-estimate the prevalence of potentially inappropriate prescribing.
Unlike previous large-scale studies that assessed the prevalence of PIMs, in 2010, Garfinkel et al. [21] conducted an interventional cohort study of 70 consecutive elderly patients which assessed the feasibility of ceasing PIMs based on a "good palliative-geriatric practice (GP-GP)" algorithm. Using the algorithm, medications in 64 patients $(91 \%)$ were discontinued. Following the discontinuation of PIMs, all participants were monitored for any change in symptoms, signs, or relevant laboratory and diagnostic tests, with follow-up assessments performed in all patients every 3 to 6 months. The results indicated that no significant adverse reactions were reported and that $84 \%$ of patients reported an improvement in health. The algorithm was heavily dependent on an experienced physician's assessment, as the two criteria for ceasing a medication were "Indication seems valid and relevant in this patient's age group and disability level" and "Do the known possible adverse reactions of the drug outweigh possible benefit in old, disabled patients?" [21]. The study recruited patients referred by their family physician or family; however, patients with advanced disease (cancer or noncancer) in whom the initial estimate of life expectancy was less than 3 months were excluded.

The second study in 2011 by Gallagher et al. [10] is the only RCT to date evaluating the outcomes of ceasing PIMs in the geriatric population in terms of prescribing appropriateness. The study randomized 400 hospitalized patients aged $\geq 65$ years to receive either the usual pharmaceutical care (control) or screening with STOPP/START Criteria with recommendations provided to their attending physicians (intervention). The Medication Appropriateness Index (MAI) and Assessment of Underutilization (AOU) index were used to assess prescribing appropriateness, both at the time of discharge and for 6 months after discharge. A total of 183 recommendations were made to the attending physicians for 111 patients (58.4\%) in the intervention group, of which, 101 (91\%) of the STOPP recommendations were accepted and the prescriptions were changed accordingly. At the study's endpoint, 135 (71.1\%) of intervention patients and $68(35.4 \%)$ of control patients had lower MAI scores at discharge than at admission demonstrating an absolute risk reduction of $35.7 \%$ as well as an absolute risk reduction in AOU of $21.2 \%$.

\section{Discussion}

The literature search identified numerous, high-impact publications describing the incidence of polypharmacy and the proportion of geriatric patients prescribed PIMs [10, 15, 17, 19-21]; however, in cancer patients, there are only three retrospective and cross-sectional, small-scale trials [1, 13, 14]. In these trials, there was a lack of consensus guidelines for assessing PIMs, instead using the authors, or a medical officer's clinical opinion. In contrast, the evidence in geriatric 
care showed many useful tools for assessing PIMs in this population. In addition, no interventional, follow-up or randomized controlled trials have been performed in palliative cancer patients, demonstrating that while PIMs exist in the population, the benefits of ceasing these medications is only theoretical.

As seen in Table 1, the study size of trials in cancer care are small when compared to that in geriatric care, with the largest retrospective trial in cancer evaluating 372 patients [13] compared to the 445,900 patients by Nyborg et al. [19]. This lack of participants could contribute to the inconsistent results between the cancer trials; for example, Reichelmann et al. found $22 \%$ of patients with at least one PIM [13], compared to the $95 \%$ found by Todd et al. [14]. The study design may have also contributed to these results, as there was a lack of a clear, standardized algorithm or guideline used for the identification of PIMs. In addition, Riechelmann et al. excluded patients still receiving cancer-directed therapy, which many palliative patients receive for quality of life purposes and may have contributed to an increased number of PIMs in these patients. Because the data from Reichelmann et al. was collected retrospectively without the ability to cross reference patient observations, the authors were not able to assess the use of antihypertensives or hypoglycemic medications used by patients [13], which may have also contributed to the lesser proportion of PIMs identified.

Another inconsistency observed between studies is the setting in which the patients were assessed. Both studies by Gallagher et al. [10, 20] assessed patients in an inpatient setting; however, palliative and hospice patients were excluded. In contrast, the other outlined studies in geriatric patients were in an outpatient setting. This difference in settings could contribute to a wide variation of proportions of patients with at least one PIM seen between the studies in geriatric patients. Of the three studies in cancer care, all were set in an outpatient setting; therefore, a gap in knowledge still exists in regard to PIMs in palliative inpatients and hospice patients, in which ADRs and pill burden are equally concerning.

There were no interventional studies identified that examined the patient outcomes of deprescribing or ceasing the PIMs in cancer. Therefore, it can only be hypothesized that the patient would benefit from ceasing a PIM, as a consequence of a decreased pill burden and medication-associated cost, an assumption that may not hold true. However, Garfinkel et al. clearly showed that ceasing PIMs in geriatric patients lead to an improvement in health with no major adverse effects reported [21]. However, as patients with malignancies were excluded, the result cannot be extrapolated to palliative cancer patients. Gallagher et al. demonstrated that by using a guideline (the STOPP/START Criteria) to reduce PIMs in patients admitted to hospital, an absolute risk reduction in MAI was achieved compared to those patients with regular hospital care [10]. This demonstrates the importance and benefits of defined guidelines; however, as terminal illness was an exclusion criteria, the guideline would require further evaluation in palliative cancer patients.

A number of guidelines were identified in the literature to identify PIMs in geriatric medicine; however, most either used, or were based on, Beers Criteria or STOPP/START. The updated Beers Criteria consists of an explicit list of medications, dosages, and durations of therapy that should be avoided in patients 65 years and older [11]. Similarly, the STOPP Criteria comprises of 65 indicators that relate primarily to important drug-drug and drug-disease interactions as well as therapeutic duplication [10]. An advantage of these guidelines is that patients' PIMs can be identified effectively based on an evidence-based approach by junior physicians or pharmacists, rather than a panel of experienced physicians. However, unlike the geriatric population, palliative cancer patients often experience distressing symptoms due to their malignancy and commonly receive numerous appropriate medications to treat complications from cancer-related treatment, cancerrelated symptoms, and comorbid illnesses [12] such as pain, anorexia, fatigue, anxiety, constipation, dyspnea, and depression [22]. As both Beers and STOPP Criteria are designed for classifying non-appropriate medications in the elderly and deem medication classes such as NSAIDs and benzodiazepines as inappropriate, there are limitations for their use in palliative cancer care.

The good palliative-geriatric practice guideline used by Garfinkel et al. [21] does show promise for palliative cancer patients as the classification of PIMs is based on its use at an individual patient level. However, this is also a limitation of the guideline, as it requires a skilled physician to use the algorithm. As malignancy was an exclusion criterion of the study, the evidence for the use of the GP-GP in an oncology setting requires evaluation. Fede et al. did develop and use a 5point algorithm to guide the identification of PIMs [1]. The algorithm is designed for palliative cancer patients, and takes into account medications that provide a limited benefit in this group such as statins and antihypertensives. However, some criteria are quite broad, and an experienced physician is necessary to use the guideline. The 5-point algorithm uses the theory that in patients with relatively short life spans, any benefit conferred by stringent "treat-to-target" regimens may be offset by treatment harm [23]. An example of this is statins, which Fede et al. included, as it has been shown that $30 \%$ of cancer patients have had a statin dispensed within 1 month of their death [24].

Other than the patient benefits implicated, the deprescribing of PIMs has the potential to reduce health care expenditure significantly [3]. Studies of cost analysis have shown that the incidence of PIMs contributes a significant burden to the healthcare system. An Irish study in 2010 showed that PIMs in the population contributed to $9 \%$ of 
the total pharmaceutical expenditure in 2007 estimated to be over 45 million Euros [3].

\section{Future directions}

Deprescribing is an important area of palliative research as all medications have potential side effects, contribute to a pill burden, and have costs to both the patient and their community. It could be argued that for palliative patients, all medications are assumed inappropriate until they improve symptom control or quality of life. The lack of evidence for many drugs in this patient group is a problem. However, before the widespread introduction of programs designed to reduce medication use in palliative care occurs, quantitative data on health outcomes are required. As a start, decision tools, such as those described by Terrell et al. in an elderly population could be adapted for use in palliative care with measured outcome data [25]. Recent advances in interventional electronic prescribing systems also show a great potential to assist deprescribing in cancer care and would enable the efficient collation of required data.

In conclusion, current data suggests that PIMs are common in palliative cancer patients; however, this review has identified a lack of criteria to easily identify PIMs in this patient group. Additionally, there are no studies measuring the patient outcomes of ceasing PIMs in palliative cancer patients. Further research is warranted to establish clear guidelines for the identification of PIMs in palliative cancer patients, as well as interventional studies assessing the outcomes of ceasing PIMs in these patients.

Disclosures None.

\section{References}

1. Fede A, Miranda M, Antonangelo D, Trevizan L, Schaffhausser H, Hamermesz B, Zimmermann C, Del Giglio A, Riechelmann RP (2011) Use of unnecessary medications by patients with advanced cancer: cross-sectional survey. Support Care Cancer 19(9):13131318. doi:10.1007/s00520-010-0947-1

2. Stevenson J, Abernethy AP, Miller C, Currow DC (2004) Managing comorbidities in patients at the end of life. BMJ 329(7471):909-912. doi:10.1136/bmj.329.7471.909

3. Cahir C, Fahey T, Teeling M, Teljeur C, Feely J, Bennett K (2010) Potentially inappropriate prescribing and cost outcomes for older people: a national population study. Br J Clin Pharmacol 69(5): 543-552. doi:10.1111/j.1365-2125.2010.03628.x

4. Lees J, Chan A (2011) Polypharmacy in elderly patients with cancer: clinical implications and management. Lancet Oncol 12(13):12491257. doi:10.1016/S1470-2045(11)70040-7

5. Gnjidic D, Le Couteur DG, Kouladjian L, Hilmer SN (2012) Deprescribing trials: methods to reduce polypharmacy and the impact on prescribing and clinical outcomes. Clin Geriatr Med 28(2):237-253. doi:10.1016/j.cger.2012.01.006

6. Reeve E, Shakib S, Hendrix I, Roberts MS, Wiese MD (2013) Development and validation of the patients' attitudes towards deprescribing (PATD) questionnaire. Int J Clin Pharm 35(1):51-56. doi:10.1007/s11096-012-9704-5

7. Holmes HM, Hayley DC, Alexander GC, Sachs GA (2006) Reconsidering medication appropriateness for patients late in life. Arch Intern Med 166(6):605-609. doi:10.1001/archinte.166.6.605

8. Riechelmann RP, Tannock IF, Wang L, Saad ED, Taback NA, Krzyzanowska MK (2007) Potential drug interactions and duplicate prescriptions among cancer patients. J Natl Cancer Inst 99(8):592600. doi:10.1093/jnci/djk130

9. Beers MH, Ouslander JG, Rollingher I, Reuben DB, Brooks J, Beck JC (1991) Explicit criteria for determining inappropriate medication use in nursing home residents. UCLA Division of Geriatric Medicine. Arch Intern Med 151(9):1825-1832

10. Gallagher PF, O'Connor MN, O'Mahony D (2011) Prevention of potentially inappropriate prescribing for elderly patients: a randomized controlled trial using STOP/START criteria. Clin Pharmacol Ther 89(6):845-854. doi:10.1038/clpt.2011.44

11. American Geriatrics Society updated Beers Criteria for potentially inappropriate medication use in older adults (2012) J Am Geriatr Soc 60(4):616-631. doi:10.1111/j.1532-5415.2012.03923.x

12. Riechelmann RP, Krzyzanowska MK, O’Carroll A, Zimmermann C (2007) Symptom and medication profiles among cancer patients attending a palliative care clinic. Support Care Cancer 15(12): 1407-1412. doi:10.1007/s00520-007-0253-8

13. Riechelmann RP, Krzyzanowska MK, Zimmermann C (2009) Futile medication use in terminally ill cancer patients. Support Care Cancer 17(6):745-748. doi:10.1007/s00520-008-0541-y

14. Todd A, Williamson S, Husband A, Baqir W, Mahony M (2013) Patients with advanced lung cancer: is there scope to discontinue inappropriate medication? Int J Clin Pharm 35(2):181-184. doi:10. 1007/s11096-012-9731-2

15. Buck MD, Atreja A, Brunker CP, Jain A, Suh TT, Palmer RM, Dorr DA, Harris CM, Wilcox AB (2009) Potentially inappropriate medication prescribing in outpatient practices: prevalence and patient characteristics based on electronic health records. Am J Geriatr Pharmacother 7(2):84-92. doi: 10.1016/j.amjopharm.2009.03.001

16. Zhan C, Sangl J, Bierman AS, Miller MR, Friedman B, Wickizer SW, Meyer GS (2001) Potentially inappropriate medication use in the community-dwelling elderly: findings from the 1996 Medical Expenditure Panel Survey. JAMA 286(22):2823-2829

17. Bongue B, Laroche ML, Gutton S, Colvez A, Gueguen R, Moulin JJ, Merle L (2011) Potentially inappropriate drug prescription in the elderly in France: a population-based study from the French National Insurance Healthcare system. Eur J Clin Pharmacol 67(12):1291-1299. doi:10.1007/s00228-011-1077-5

18. Laroche ML, Charmes JP, Merle L (2007) Potentially inappropriate medications in the elderly: a French consensus panel list. Eur J Clin Pharmacol 63(8):725-731. doi:10.1007/s00228007-0324-2

19. Nyborg G, Straand J, Brekke M (2012) Inappropriate prescribing for the elderly - a modern epidemic? Eur J Clin Pharmacol 68(7):10851094. doi:10.1007/s00228-012-1223-8

20. Gallagher P, Lang PO, Cherubini A, Topinkova E, CruzJentoft A, Montero Errasquin B, Madlova P, Gasperini B, Baeyens H, Baeyens JP, Michel JP, O’Mahony D (2011) Prevalence of potentially inappropriate prescribing in an acutely ill population of older patients admitted to six European hospitals. Eur J Clin Pharmacol 67(11):1175-1188. doi:10.1007/s00228-011-1061-0

21. Garfinkel D, Mangin D (2010) Feasibility study of a systematic approach for discontinuation of multiple medications in older adults: 
addressing polypharmacy. Arch Intern Med 170(18):1648-1654. doi: 10.1001/archinternmed.2010.355

22. Walsh D, Donnelly S, Rybicki L (2000) The symptoms of advanced cancer: relationship to age, gender, and performance status in 1,000 patients. Support Care Cancer 8(3):175-179

23. Scott IA, Gray LC, Martin JH, Mitchell CA (2012) Minimizing inappropriate medications in older populations: a 10-step conceptual framework. Am J Med 125(6):529-537 e524. doi:10.1016/j.amjmed. 2011.09.021
24. Stavrou EP, Buckley N, Olivier J, Pearson SA (2012) Discontinuation of statin therapy in older people: does a cancer diagnosis make a difference? An observational cohort study using data linkage. BMJ Open. 2(3). doi:10.1136/bmjopen-2012-000880

25. Terrell KM, Perkins AJ, Dexter PR, Hui SL, Callahan CM, Miller DK (2009) Computerized decision support to reduce potentially inappropriate prescribing to older emergency department patients: a randomized, controlled trial. J Am Geriatr Soc 57(8):1388-1394. doi: 10.1111/j.1532-5415.2009.02352.x 